Rabaska

Revue d'ethnologie de l'Amérique française

\title{
Une famille porteuse de traditions : les Morneau de Baie-des-Rochers
}

\section{Jean-Benoît Guérin-Dubé}

Volume 13, 2015

Présence de Marius Barbeau : l'invention du terrain en Amérique

française. Autour d'un legs centenaire (1914-2014)

URI : https://id.erudit.org/iderudit/1033747ar

DOI : https://doi.org/10.7202/1033747ar

Aller au sommaire du numéro

Éditeur(s)

Société québécoise d'ethnologie

ISSN

1703-7433 (imprimé)

1916-7350 (numérique)

Découvrir la revue

Citer cet article

Guérin-Dubé, J.-B. (2015). Une famille porteuse de traditions : les Morneau de Baie-des-Rochers. Rabaska, 13, 37-41. https://doi.org/10.7202/1033747ar d'utilisation que vous pouvez consulter en ligne. 


\title{
Une famille porteuse de traditions : les Morneau de Baie-des-Rochers
}

\author{
JEAN-BENOÎT GuÉRIN-DUBÉ \\ Université du Québec à Chicoutimi
}

C'est sur les traces de Marius Barbeau que s'entame cette magnifique épopée qu'est la cueillette de traditions. Dans un petit hameau de Charlevoix, à Baie-des-Rochers, un lieu où Barbeau n'a jamais mis les pieds ${ }^{1}$, on a fait une découverte d'envergure. Baie-des-Rochers deviendra dès lors un lieu d'enquête privilégié pour deux grands hommes. Dans un premier temps, ce sera l'abbé Félix-Antoine Savard qui découvrira certains porteurs de traditions, plus particulièrement la famille Morneau. Par la suite, $\mathrm{M}^{\mathrm{gr}}$ Savard $^{2}$ poursuivra son travail avec l'aide de Luc Lacourcière, ce qui permettra surtout de sauvegarder puis de transmettre une partie non négligeable du folklore québécois.

Pour comprendre comment ce terrain d'enquête deviendra important au cours des années 1940, il est toutefois nécessaire de donner un aperçu de Baie-des-Rochers.

Géographiquement parlant, Baie-des-Rochers est situé à environ quinze kilomètres à l'est du village de Saint-Siméon. À l'entrée de la baie, il y a deux lieux d'importance : au nord, Cap du Nid aux Corbeaux, montagneux et escarpé ; au sud, l'Anse-de-Sable qui deviendra le futur lieu d'établissement du chalet du curé Savard. Il y a aussi une île en plein milieu de la baie. Et comme de nombreux sites, voire une majorité, qui bordent le fleuve SaintLaurent, c'est Samuel de Champlain qui nomma cet endroit « Anse aux

1. Serge Gauthier, Charlevoix ou la création d'une région folklorique. Étude du discours de folkloristes québécois (1916-1980), Québec, Presses de l’Université Laval, 2006, XIII-208 p.

2. L'abbé Félix-Antoine Savard (1896-1982) est nommé vicaire à Sainte-Agnès en 1927, puis à La Malbaie en 1929, enfin curé fondateur de la paroisse de Clermont en 1931. À la suite de la publication de son roman Menaud maittre draveur (1937), il fait la rencontre de Luc Lacourcière avec qui il fonde les Archives de folklore à l'Université Laval en 1944. Ce n'est qu'en 1950 qu'il deviendra prélat domestique et portera le titre de $\mathrm{M}^{\mathrm{gr}}$ Savard par lequel on le désigne depuis. 
Rochers », en 1626. « Baye des Rochers » deviendra le nom usuel à partir d'une carte du père Laure en $1731^{3}$.

Selon la légende locale, les premiers habitants de l'endroit seraient Jean Savard et Félicité Therrien qui seraient arrivés en canot dans les années $1820^{4}$. Toutefois, le premier résultat d'un développement de population et surtout d'une industrie apparaît en 1838 par l'érection d'un moulin à scie ${ }^{5}$. Baie-desRochers sera rattachée officiellement à la paroisse de Saint-Siméon quand le canton Callières sera annexé en décembre $1899^{6}$. Malheureusement, il n'y a pas de chiffres clairs sur la population locale à l'époque. Pour la période dont il est question ici, les recensements ecclésiastiques de Saint-Siméon sont d'une grande aide. En août 1933, la population s'élevait à 155 âmes alors qu'en 1968, elle s'élevait à un peu plus de 180 âmes?

Par ces particularités, Baie-des-Rochers devient rapidement un lieu d'enquête potentiel. Le caractère isolé de l'endroit, à l'époque où Savard et Lacourcière feront leurs enquêtes, permet de croire que le folklore y est encore " inchangé ». Le lieu semble aussi posséder cette " pureté » que recherchait tant Barbeau lors de ses enquêtes ${ }^{8}$. Baie-des-Rochers ne possède pas les éléments de base qui définissaient un village à l'époque. Il n'y avait pas de curé résidant, ni de membres de professions libérales. La population possédait tout de même une petite chapelle où les messes furent célébrées dès 1903. Le caractère insulaire incontournable de cet endroit est ce qui attire le plus Félix-Antoine Savard9.

La rencontre entre la famille Morneau et l'abbé Savard eut lieu à la fin des années 1930. Les Morneau proviennent du nord-ouest de la France et leurs ancêtres seraient François Morneau et une certaine Marie Mornet. La date de leur établissement au Canada demeure approximative, mais André Morneau sera le premier dont on retrouvera la trace. Son fils Germain aura une nombreuse descendance qui s'établira surtout sur la Côte-Nord aux alentours de Tadoussac, de Sacré-Cœur et des Escoumins ${ }^{10}$. Il n'y aura que Philéas qui choisira Charlevoix, et plus particulièrement Baie-des-Rochers, comme lieu d'établissement. C'est là que l'abbé Savard fit la connaissance

3. Commission de toponymie du Québec.

4. "Saint-Siméon », Revue d'histoire de Charlevoix, hors-série n 3, Société d'histoire de Charlevoix, 1994.

5. Louis Pelletier, La Seigneurie de Mount Murray, Québec, Éditions du Septentrion, 2008, $398 \mathrm{p}$.

6. Archives municipales de Saint-Siméon, 1869-2014, " Procès-verbaux de la municipalité », Saint-Siméon, Charlevoix.

7. Archives ecclésiastiques de la Paroisse de Saint-Siméon, « Prônes », dépouillement de 19271973.

8. Serge Gauthier, op. cit.

9. Ibid.

10. Frère Éloi-Gérard, Recueil de généalogies des comtés de Charlevoix et Saguenay depuis l'origine jusqu'à 1939, La Malbaie, 1941. 


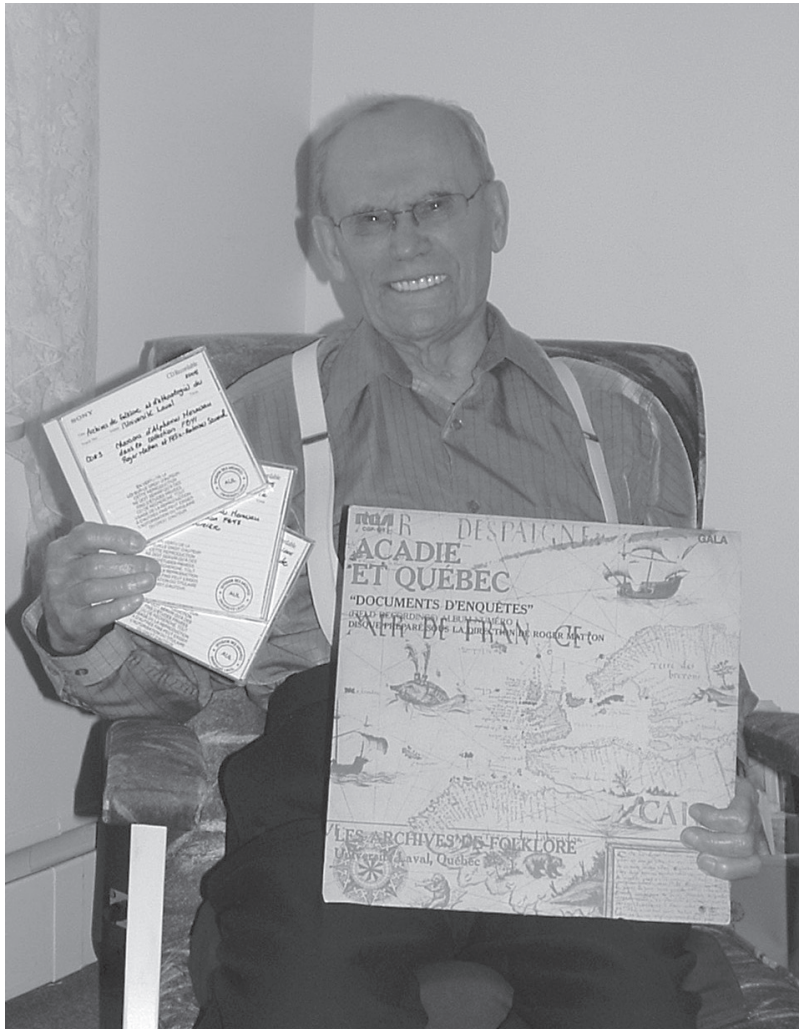

Alphonse Morneau tenant le disque Acadie et Québec et les enregistrements qu'il a confiés aux enquêteurs, vers 2008.

Source : collection Guillaume Savard : photo d'Élise Tremblay.

de cette famille. Désirant faire le tour de la baie, Philéas, le patriarche de la famille, s'offrira pour réaliser cette excursion ${ }^{11}$.

Pour le curé Savard, la visite de la Baie-des-Rochers sera un véritable coup de foudre, surtout lorsqu'il découvrira l'Anse-de-Sable. Après presqu'une année complète sans nouvelles, l'abbé envoie une lettre à la famille Morneau demandant de lui construire un chalet à l'Anse-de-Sable, accompagné des plans dessinés par un architecte de Québec ${ }^{12}$. Philéas Morneau, son fils Alphonse et d'autres membres de la famille élargie participeront à la construction du chalet en plus d'amis de la famille. Et c'est durant cette construction que le prêtre verra à quel point Baie-des-Rochers est un lieu privilégié pour le folklore québécois.

11. Guillaume Savard, «Baie-des-Rochers : Refuge de 1'écrivain Félix-Antoine Savard », Revue d'histoire de Charlevoix, Société d'histoire de Charlevoix, n 20, novembre 1994.

12. Guillaume Savard, "Mon grand-père, le chanteur », Revue d'histoire de Charlevoix, Société d'histoire de Charlevoix, n 20 , novembre 1994. 
Lors d'une de ses nombreuses présences pendant la construction du chalet, l'abbé Savard entendit fredonner. C'était Alphonse, fils de Philéas, travaillant sur la couverture qui émettait ces sons. À l'exemple de sa mère, Alphonse chantait en travaillant. Cet environnement folklorique lui était familier depuis son enfance. C'est de Rose-Anne Bouchard, sa mère, qu'Alphonse apprit toutes ces chansons, car elle passait ses journées à fredonner et à chanter pendant qu'elle filait au rouet, tricotait et s'adonnait à toutes ses autres activités ${ }^{13}$. Quelle ne fut pas la surprise du religieux de percevoir, en cet Alphonse Morneau, un véritable vecteur du folklore québécois, de la tradition, de l'histoire.

Suite à cet épisode de la construction de son chalet, l'abbé fit plus ample connaissance avec la famille Morneau. Il vit bien vite que la famille entière était empreinte de tradition, surtout en ce qui a trait aux chansons folkloriques et aux contes. Lorsqu'il s'attarda à la matriarche Rose-Anne, il prit réellement conscience qu'il fallait impérativement sauvegarder et transmettre ce savoir qui continuait de survivre dans ce lieu loin de tout. Personne ne sait si Rose-Anne Bouchard pouvait réellement fredonner près de 500 chansons, mais il n'en demeure pas moins que son répertoire était vaste et celui de son fils Alphonse l'était tout autant. On venait ainsi de repérer chez cette famille Morneau des porteurs de traditions.

C'est en 1941 que la cueillette folklorique commencera officiellement à Baie-des-Rochers ${ }^{14}$. L'abbé Savard y viendra accompagné de Luc Lacourcière. Suivant la méthode de Marius Barbeau, Savard et Lacourcière s'empresseront de faire des enregistrements, en commençant d'abord par la voix d'Alphonse. Grâce à ces investigations, toutes les chansons familiales seront finalement sauvegardées aux Archives de folklore de l'Université Laval. Certains titres que Lacourcière ne croyait jamais entendre, comme les Écoliers de Pontoise, $\mathrm{y}$ furent enregistrés ${ }^{15}$. Même que cela a permis à Alphonse Morneau de « [se] faire connaître non seulement au Canada français, mais jusqu'aux États-Unis et dans la lointaine Europe ${ }^{16} \gg$. On recensa encore plusieurs contes, mais sur ce point, les informations manquent pour donner de plus amples descriptions de ce qui a été sauvegardé.

L'apport des Morneau a donc été fort important sur plusieurs aspects du folklore. Bien entendu, le folklore étant en perpétuelle évolution, on a noté certaines divergences dans l'interprétation entre la version entendue jadis et celle enregistrée par les Morneau, illustrant la richesse qu'apporte la tradi-

\section{Ibid.}

14. Ibid.

15. Aurélien Boivin, «Une entrevue avec Luc Lacourcière », Québec français, n² 27, 1997, p. 27 31); aussi en ligne : http://id.erudit.org/iderudit/56664ac, consulté le 31 juillet 2014.

16. Francine Brunel-Reeves, " "L'Homme aux 400 chansons n'est plus". Alphonse Morneau (1914-2010) », Rabaska, vol. 8, 2010, p. 165-177. 
tion, reflet de la vie qui continue et qui se transforme. Encore aujourd'hui, la tradition familiale se poursuit au niveau musical par Guillaume Savard, petit-fils d'Alphonse. Certains avaient dit à Barbeau qu'un jour on le remercierait pour ce qu'il a fait. Je me dois de le faire ici : merci ! Car, sans lui, le folklore, notamment la chanson, n'aurait pas le renom qu'on lui accorde de nos jours. Et, s'il n'y avait pas eu de Félix-Antoine Savard et de Luc Lacourcière à l'affût des traditions, on n'aurait jamais reconnu des familles comme les Morneau, et de nombreuses autres rencontrées par la suite, comme des « porteuses de traditions ».

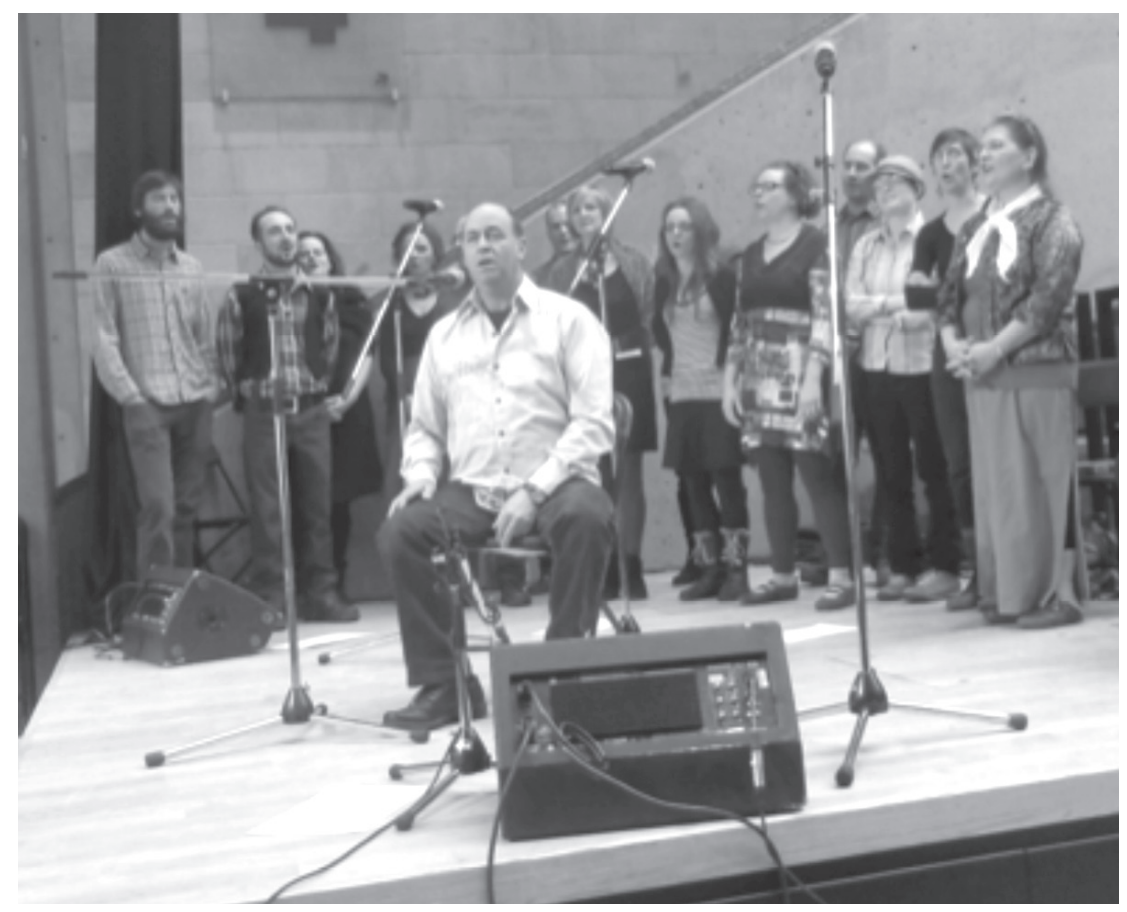

Guillaume Savard, conteur et chanteur, petit-fils d'Alphonse Morneau, 24 janvier 2015.

Source : collection Guillaume Savard (guillaumesavard.wordpress.com). 